\title{
Comparison of T1 mapping techniques for ECV quantification. Histological validation and reproducibility of ShMOLLI versus multibreath-hold T1 quantification equilibrium contrast CMR
}

\author{
Marianna Fontana ${ }^{1,2}$, Steve K White ${ }^{1,2}$, Sanjay M Banypersad ${ }^{1,2}$, Daniel M Sado ${ }^{1,2}$, Viviana Maestrini ${ }^{1,2}$,
} Andrew S Flett ${ }^{1,2}$, Stefan K Piechnik ${ }^{3}$, Stefan Neubauer ${ }^{3}$, Neil Roberts ${ }^{1}$ and James C Moon ${ }^{1,2^{*}}$

\begin{abstract}
Background: Myocardial extracellular volume (ECV) is elevated in fibrosis or infiltration and can be quantified by measuring the haematocrit with pre and post contrast $\mathrm{T} 1$ at sufficient contrast equilibrium. Equilibrium CMR (EQ-CMR), using a bolus-infusion protocol, has been shown to provide robust measurements of ECV using a multibreath-hold T1 pulse sequence. Newer, faster sequences for T1 mapping promise whole heart coverage and improved clinical utility, but have not been validated.

Methods: Multibreathhold T1 quantification with heart rate correction and single breath-hold T1 mapping using Shortened Modified Look-Locker Inversion recovery (ShMOLLI) were used in equilibrium contrast CMR to generate ECV values and compared in 3 ways.

Firstly, both techniques were compared in a spectrum of disease with variable ECV expansion ( $n=100$, 50 healthy volunteers, 12 patients with hypertrophic cardiomyopathy, 18 with severe aortic stenosis, 20 with amyloid). Secondly, both techniques were correlated to human histological collagen volume fraction (CVF\%, $n=18$, severe aortic stenosis biopsies). Thirdly, an assessment of test:retest reproducibility of the 2 CMR techniques was performed 1 week apart in individuals with widely different $E C V s$ ( $n=10$ healthy volunteers, $n=7$ amyloid patients).

Results: More patients were able to perform ShMOLLI than the multibreath-hold technique ( $6 \%$ unable to breath-hold). ECV calculated by multibreath-hold T1 and ShMOLLI showed strong correlation $\left(r^{2}=0.892\right)$, little bias (bias $-2.2 \%, 95 \% \mathrm{Cl}$ $-8.9 \%$ to $4.6 \%$ ) and good agreement (ICC 0.922 , range 0.802 to $0.961, p<0.0001$ ). ECV correlated with histological CVF\% by multibreath-hold ECV $\left(r^{2}=0.589\right)$ but better by ShMOLLI ECV $\left(r^{2}=0.685\right)$. Inter-study reproducibility demonstrated that ShMOLLI ECV trended towards greater reproducibility than the multibreath-hold ECV, although this did not reach statistical significance $(95 \% \mathrm{Cl}-4.9 \%$ to $5.4 \%$ versus $95 \% \mathrm{Cl}-6.4 \%$ to $7.3 \%$ respectively, $\mathrm{p}=0.21)$.

Conclusions: ECV quantification by single breath-hold ShMOLLI T1 mapping can measure ECV by EQ-CMR across the spectrum of interstitial expansion. It is procedurally better tolerated, slightly more reproducible and better correlates with histology compared to the older multibreath-hold FLASH techniques.
\end{abstract}

Keywords: Interstitial space, Fibrosis, CMR

\footnotetext{
* Correspondence: james.moon@uclh.nhs.uk

${ }^{1}$ The Heart Hospital, 16-18 Westmoreland Street, London W1G 8PH, United Kingdom

${ }^{2}$ Institute of Cardiovascular Science, University College London, London WC1E 6BT, United Kingdom

Full list of author information is available at the end of the article
} 


\section{Background}

The myocardial extracellular space is expanded by focal fibrosis [1], diffuse fibrosis [2-6] or infiltration, such as amyloidosis [7]. It can be measured non-invasively by cardiovascular magnetic resonance (CMR) using pre and post contrast $\mathrm{T} 1$ relaxation times of blood and myocardium (the latter at sufficient contrast equilibrium) with correction for the blood volume of distribution via the haematocrit $[1,2,8]$. A number of T1 measurement techniques exist including multibreath-hold techniques such fast low angle single shot inversion recovery ("multibreathhold FLASH-IR"). Here the sequence is performed at increasing inversion times to generate $\mathrm{T} 1$ recovery curves and heart rate correction $[9,10]$. Newer, faster sequences such as MOLLI (Modified Look-Locker Inversion recovery) [11] perform IR measurements in a single breath-hold. A recent evolution of MOLLI, the Shortened-MOLLI (ShMOLLI) [12] improves clinical utility with a shorter breath-hold and immediate map reconstruction directly on the scanner. ECV measurements have been performed and validated using MOLLI for bolus-only protocols. Equilibrium contrast CMR (EQ-CMR) with single breath-hold sequences has not yet been validated for ECV assessment. ECV mapping with such sequences would be a significant technical advance, being easier for patients with shorter breathholds (either shorter scans or whole heart coverage) and easier quantification.

We hypothesised that ECV mapping using ShMOLLI would be superior to the multibreath-hold FLASH-IR technique. This was assessed in three ways: firstly, to determine any bias in ECV between the two techniques. Secondly, we compared both CMR techniques with histological collagen volume fraction (CVF\%). Finally, we assessed the reproducibility of both CMR techniques. For equivalence of contrast conditions between the two techniques, we used the primed infusion technique, equilibrium contrast CMR.

\section{Methods \\ CMR protocol}

The research received approval from the local research ethics committee and all participants provided written informed consent. EQ-CMR was performed as described previously [9]. CMR was performed on a $1.5 \mathrm{~T}$ magnet (Avanto, Siemens Medical Solutions). Within a standard clinical scan (pilots, transverse white and black blood images, volumes, and LGE imaging) T1 measurement pre-contrast was performed using (a) FLASH-IR at increasing inversion times from 140 to $800 \mathrm{~ms}$ (or $900 \mathrm{~ms}$ if patient heart rate permitted), "multibreath-hold technique", Figure 1a and (b) ShMOLLI T1 mapping "single breath-hold technique", Figure 1b. After a bolus of Gadoterate meglumine, $(0.1 \mathrm{mmol} / \mathrm{kg}$, gadolinium-
DOTA, marketed as Dotarem (c) Guerbet S.A. France) and standard LGE imaging, at 15-minute post bolus, an infusion at a rate of $0.0011 \mathrm{mmol} / \mathrm{kg} / \mathrm{min}$ contrast (equivalent to $0.1 \mathrm{mmol} / \mathrm{kg}$ over 90 minutes) was given. The patient was typically removed from the scanner at this time. At between 45 minutes and 80 minutes post bolus, the patient was returned to the scanner, still with the infusion, and the T1 measurement repeated using both multi and single breath-hold techniques. Separate regions of interest (ROIs) were placed in all available images and recovery curve was reconstructed by fitting the relaxation formula to ROI averages. Heart rate correction was used for the multibreath-hold technique [9]. In the ShMOLLI sequence, T1 maps were generated using previously published algorithm [12]. A single ROI was drawn directly in each T1 map at the same location as the multibreath-hold technique and T1 averaged between all pixels (Figure 1b). A haematocrit was taken in all subjects. The ECV was calculated with each method as Myocardial ECV $=(1$-haematocrit $) \times\left(\Delta \mathrm{R} 1_{\text {myocardium }} /\right.$ $\left.\Delta \mathrm{R} 1_{\text {blood }}\right)$ [1]. $\mathrm{T} 1$ was measured in the basal to mid septum avoiding areas of late gadolinium enhancement, except in myocardial infarction (where the infarct zone was assessed) and amyloid (where the regions was drawn irrespective of the ill-defined presence/absence of LGE). The blood T1 was assessed in the descending aorta. All the analysis were performed blinded.

\section{Patient studies: healthy normal subjects and disease groups}

Normal subjects ( $n=50$, median age $47 \pm 17,53 \%$ male) were recruited through advertising within the hospital, university and general practitioner surgeries. All normal subjects had no history or symptoms of cardiovascular disease or diabetes. Four subjects had been prescribed statin therapy for hypercholesterolaemia (primary cardiovascular prevention), but no other normal subject was taking any cardiovascular medication. All subjects had a normal blood pressure, 12 lead electrocardiogram and clinical CMR scan.

Patients $(n=50)$ were prospectively recruited from tertiary clinical and research departments at the Heart Hospital or the National Amyloidosis Centre, Royal Free Hospital: [1] 12 patients with hypertrophic cardiomyopathy meeting the diagnostic criteria (average age $52 \pm 13$, $60 \%$ male). [2] 18 patients with severe aortic stenosis waiting for aortic valve replacement (median age: $71 \pm 10$, 72\% male). [3] 20 patients with cardiac AL amyloidosis with disease proven by non-cardiac biopsy and cardiac involvement ascertained through echocardiography, supported by a Mayo clinic classification score of 2 or 3 (average age: $60 \pm 10,75 \%$ male). Patients with atrial fibrillation or a contra-indication to contrast CMR examination were excluded from the study. 


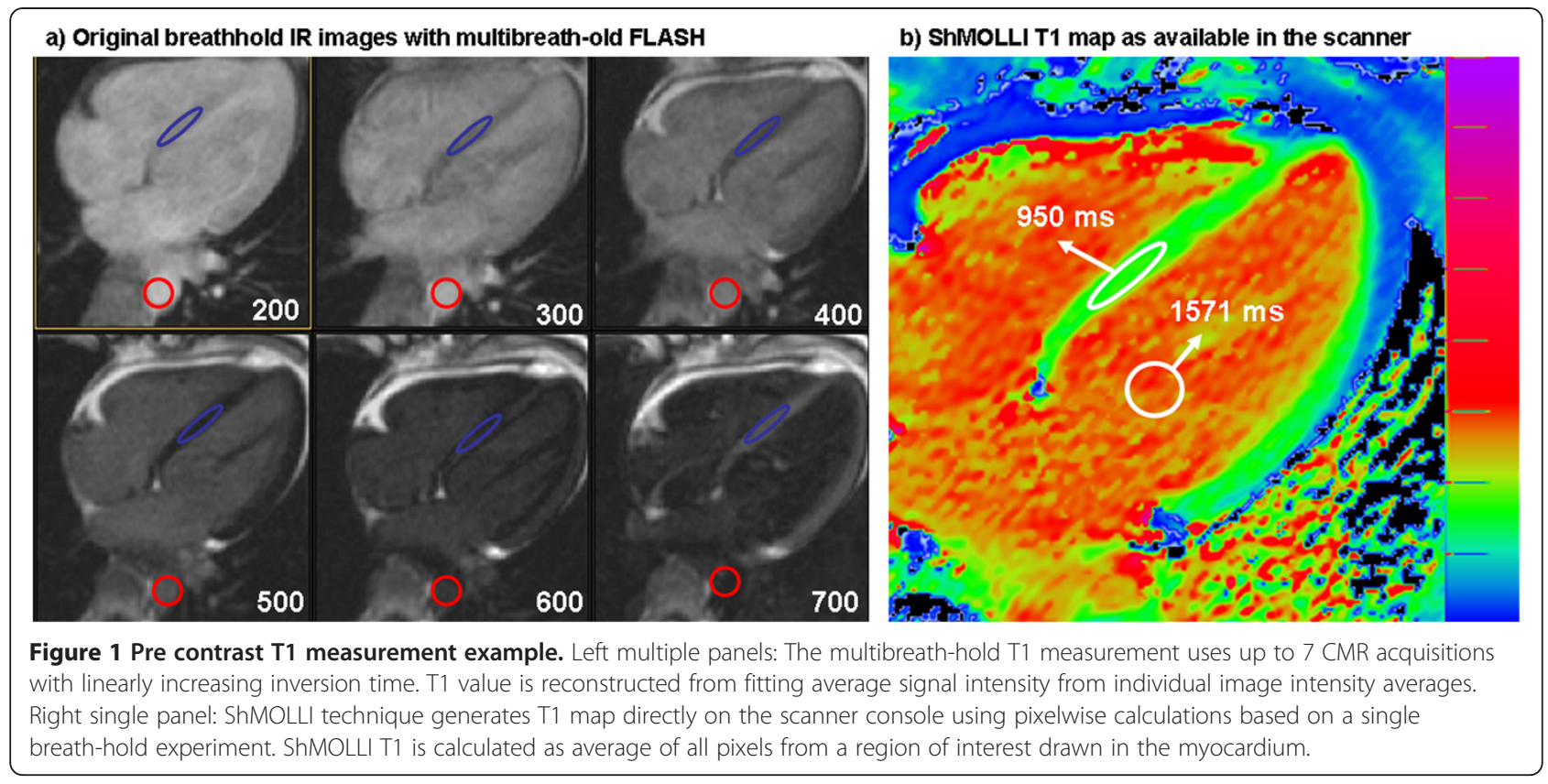

\section{Histological validation}

24 patients with severe aortic stenosis listed for surgical aortic valve replacement were studied. An intraoperative deep myocardial biopsy (Tru-Cut needle) was taken in aortic stenosis. Samples were stained and analysed for CVF\%, as previously described [9]. 6 patients were excluded from further analysis ( 2 patients had focal fibrosis detected as LGE in the basal septum; 2 patients had biopsies consisting solely of endocardial fibrosis; in 1 patient multibreathhold T1 quantification was not performed because the patient was unable to breath-hold; 1 patient had a pulmonary oedema during the scan) leaving 18 patients.

\section{Reproducibility}

For test:retest interstudy reproducibility, 10 normal subjects and 7 patients with amyloid underwent repeat scanning, one week apart. The analysis was carried out by a single observer blinded.

\section{Data analysis and statistics}

Results were analysed using SPSS (Chicago, IL, USA, version 19). ECV values were found to be normally distributed in each group (Kolmogorov-Smirnov test, p $>0.05$ for each data set) and so expressed as mean \pm standard deviation. We used Intraclass Correlation Coefficient (ICC) and Bland-Altman plot to compare ECV values from the two methods. Interstudy reproducibility for the CMR measurement of ECV was assessed by calculating the ICC and Bland Altman plots. To compare the squared difference of paired ECV measurements Wilcoxon signed rank test was used. A p value $<0.05$ was considered statistically significant.

\section{Results}

\section{ECV comparison}

No patients failed ShMOLLI acquisition. 6\% of patients were unable to perform all the 14 breath-holds required for the multibreath-hold technique. The mean ECV assessed using multibreath-hold T1 quantification and ShMOLLI T1 in normal subjects and disease groups is shown in Table 1. ECV by multibreath-hold T1 quantification and by ShMOLLI T1 mapping showed excellent correlation $\left(\mathrm{r}^{2}=0.892\right)$ and agreement across disease groups (overall ICC 0.922, 95\%CI 0.802 to 0.961 , $\mathrm{p}<0.0001$ ), Figure 2a, with little bias on Bland Altman (bias $-2.2 \%, 95 \% \mathrm{CI}-8.9 \%$ to $4.6 \%$ ), Figure $2 \mathrm{~b}$.

\section{Histological validation}

All biopsies were uneventful. The mean histological CVF of the 18 biopsies was $18 \% \pm 8 \%$ (range $7 \%$ to $40 \%$ ). There was a strong correlation between histological CVF\% and FLASH ECV $\left(r^{2}=0.589\right)$ but this was stronger with ShMOLLI ECV $\left(r^{2}=0.685\right)$ (Figure 3$)$.

Table 1 Mean ECV \pm standard deviation assessed using multibreath-old T1 quantification and ShMOLLI T1 in normal subjects and disease groups

\begin{tabular}{lll}
\hline & $\begin{array}{l}\text { Multibreath-hold } \\
\text { T1 ECV (\%) }\end{array}$ & $\begin{array}{l}\text { ShMOLLI } \\
\text { ECV (\%) }\end{array}$ \\
\hline Normal subjects $(n=50)$ & $26 \pm 3$ & $27 \pm 3$ \\
HCM $(n=12)$ & $28 \pm 4$ & $30 \pm 3$ \\
AS $(n=18)$ & $27 \pm 6$ & $31 \pm 5$ \\
Amyloid $(n=20)$ & $48 \pm 6$ & $52 \pm 7$ \\
\hline
\end{tabular}



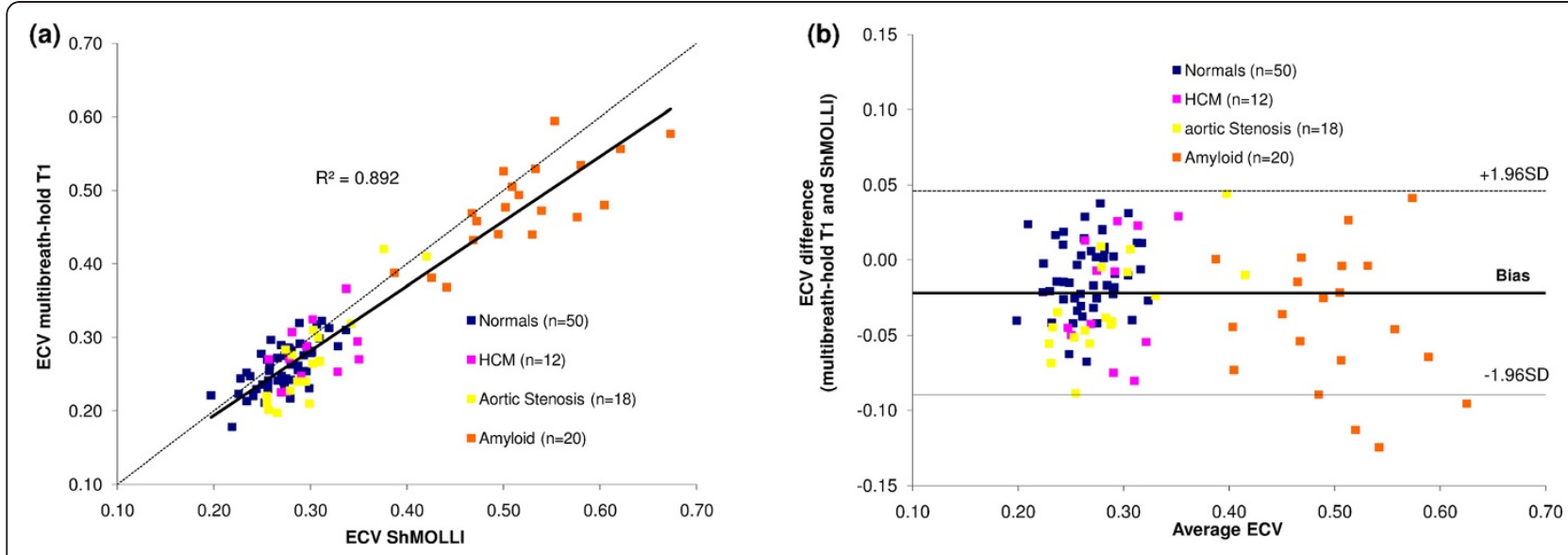

Figure 2 Sh-MOLLI and multibreath-hold ECV correlation in health and disease (panel a) and the same data plotted as a Bland-Altman analysis (panel b), showing little bias.

\section{Interstudy reproducibility}

Inter-study reproducibility demonstrated that ShMOLLI ECV was slightly more reproducible than the multibreathhold ECV, Figure 4, (ICC 982, 95\%CI 0.951-0.994 versus ICC 962, 95\%CI 0.897-0.987), with narrower confidence intervals $(95 \% \mathrm{CI}-4.9 \%$ to $5.4 \%$ versus $95 \% \mathrm{CI}-6.4 \%$ to $7.3 \%$ respectively). Neither of these reached statistical significance however, $\mathrm{P}>0.2$.

\section{Discussion}

CMR ECV quantification requires accurate and rapid T1 relaxation time calculation. We have found that ECV quantification using ShMOLLI compared to multibreath-hold T1 quantification has a greater chance of technical success on all patients, less bias over a wide range of ECV measurements, a higher correlation with histological CVF\% as well as better reproducibility. These four findings combine with key advantages of the mapping technique: a single breath-hold per T1 map, simple analysis and the potential for whole heart ECV quantification. Therefore, it is our opinion that T1 mapping technique ShMOLLI is the superior CMR technique for ECV quantification.

T1 mapping by means of the multi-breath-hold technique is one of the most commonly utilised methods for T1 quantification $[9,10]$. The main advantage of this technique is that it is not vendor specific and it has been heavily optimised by everyday clinical practice to obtain high resolution LGE images. However it also has a number of limitations. Firstly, in order to map a single cardiac slice, it requires the sequence to be run up to 9 times at increasing inversion times. The average breathhold time for each sequence is 14 seconds (longer at slower heart rates). If the patient cannot hold their breath for the duration of the sequence, artefact will appear on any one of the images. This may also occur if
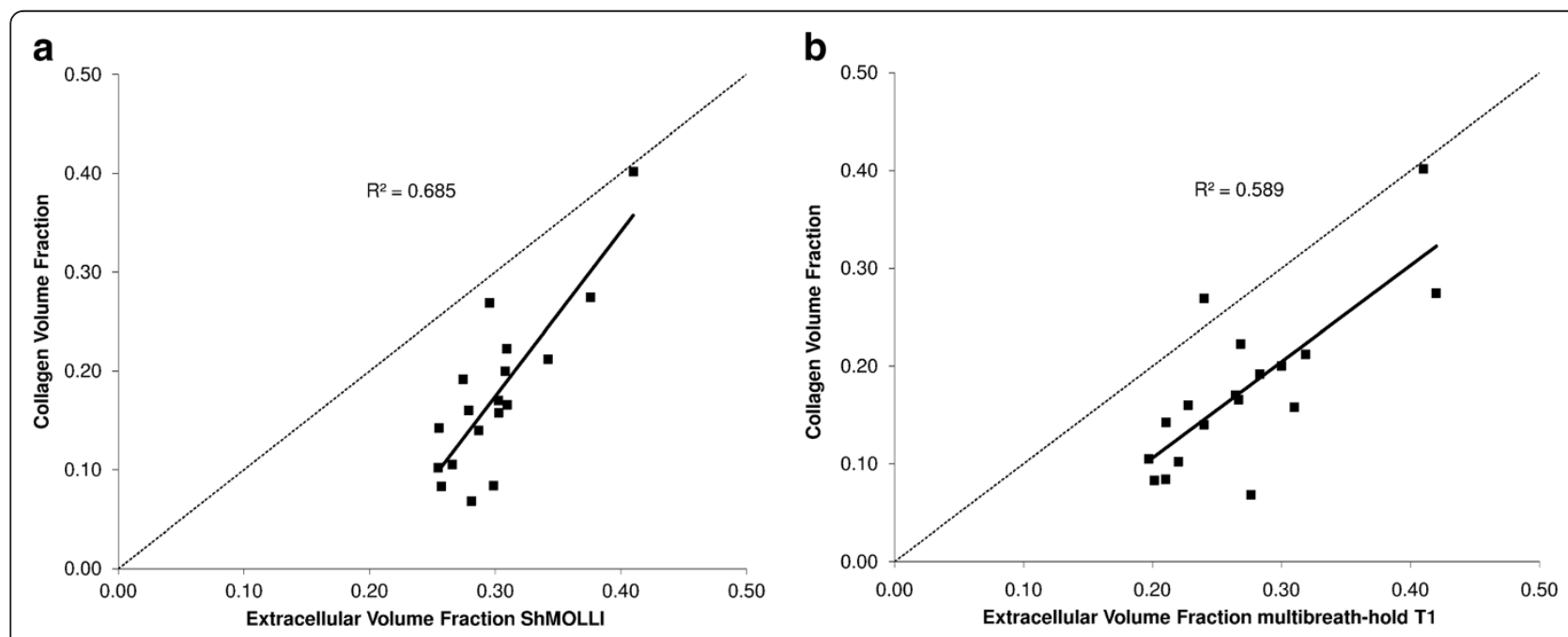

Figure 3 ECV against histological CVF\% $(n=18)$ by ShMOLLI (panel a) and multi-breath-hold (panel b). 

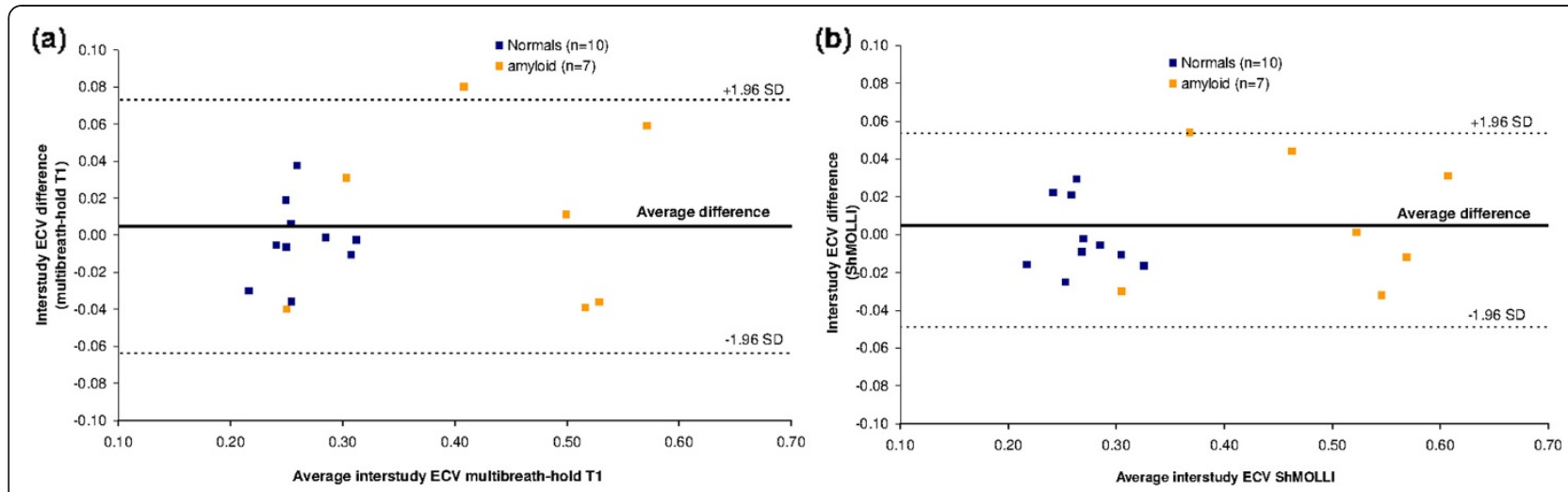

Figure 4 Bland-Altman analysis to express the intrasequence reproducibility of the myocardial T1 values using FLASH-IR (panel a) and ShMOLLI (panel b) in normal subjects (blu square) and amyloid patients (orange square). The limits of agreement are slightly narrower for ShMOLLI than multibreath-hold.

the patient has an arrhythmia. As each slice takes so many breath-holds to map, this method can not be used clinically for whole heart mapping (i.e. of multiple cardiac slices). Once the images have been obtained, the offline post processing is laborious and the results require heart rate correction for the calculated T1 relaxations times [9].

We have found that ECV quantification using ShMOLLI improves on several practical aspects compared to multibreath-hold T1 quantification. ShMOLLI imaging is characterised by lower breath-hold failure rate, less bias over a wide range of ECV, a slightly higher correlation with histological collagen volume fraction and slightly better reproducibility, although it does not reach statistical significance. Shorter acquisition times and direct T1 map calculation on the scanner [12] ensures ShMOLLI is quicker to perform and analyse with a potential to provide whole heart ECV quantification. While currently ShMOLLI is vendor specific, its predecessor MOLLI exists for more than one platform [13].

Future techniques for T1 measurement are likely to be based on mapping. Further advances may include higher resolution imaging with reduction of partial volume effects, with that incorporate motion correction and improved capabilities for measuring longer T1s [14-17]. We preferred here to re-iterate the T1 map for maximum accuracy - a step that will likely be un-necessary in future refinements. Preliminary exploration is being made of combining the two T1 maps into an ECV map using further non-rigid registration [10].

This study has limitations. We have not presented the phantom work comparing ShMOLLI and FLASH-IR T1 estimation as this was primarily an ECV clinicallyorientated paper with many of the likely confounders present only in-vivo and not detectable by phantom work. Histology correlations were lower previously described [9]. We believe this is primarily due to different population characteristics (the population examined here is older) and to a greater number of surgeons involved in the biopsy arm of this study (with associated reduced homogeneity of samples). Finally, FLASH-IR was compared to a single T1 mapping technique, ShMOLLI. MOLLI,its variants and other T1 mapping techniques such as SACHA [18] were not considered in this study.

As ECV quantification experience increases, it is likely that technology will advance to become more precise, accurate and automated. Our study represents the first such paper and demonstrates that single breath-hold ShMOLLI T1 mapping can quantify ECV by EQ-CMR across the spectrum of interstitial expansion, and that it is clinically more straightforward with improvements in reproducibility and histological correlation when compared to the older multibreath-hold FLASH techniques.

\section{Conclusion}

ECV quantification by single breath-hold ShMOLLI T1 mapping can measure ECV by EQCMR across the spectrum of interstitial expansion. It is procedurally better tolerated, slightly more reproducible and better correlates with histology compared to the older multibreathhold FLASH techniques.

\section{Competing interests}

The authors declare that they have no competing interests.

\section{Authors' contribution}

MF: led the study; co-ordinated analysis; lead writer of manuscript. SKW, SMB, DMS, VM each contributed a patient group along with the respective analysis. SKP \& SN: technical support for T1 mapping sequence/ShMOLLI development, analysis of T1 maps. ASF: blind analysis of histological data. NR: CO-ordinated the surgical support and biopsy. JCM: concept and design of study. All authors read and approved the final manuscript. 


\section{Acknowledgements}

This work was undertaken at UCLH/UCL who received a proportion of funding from the Department of Health's NIHR Biomedical Research Centres funding scheme. SN and SKP acknowledge support from the National Institute for Health Research Oxford Biomedical Research Centre Programme. JCM is funded by HEFCE. SKW, DMS, ASF are funded by the British Heart Foundation. SN also acknowledges support from the Oxford British Heart Foundation Centre of Research Excellence.

\section{Disclosures}

US patent pending 61/387,591: SKP. SYSTEMS AND METHODS FOR SHORTENED LOOK LOCKER INVERSION RECOVERY (Sh-MOLLI) CARDIAC GATED MAPPING OF T1. September 29, 2010. All rights sold exclusively to Siemens Medical. All the other authors declare that they have no competing interests.

\section{Author details}

${ }^{1}$ The Heart Hospital, 16-18 Westmoreland Street, London W1G 8PH, United Kingdom. Institute of Cardiovascular Science, University College London, London WC1E 6BT, United Kingdom. ${ }^{3}$ Department of Cardiovascular Medicine Oxford Centre for Clinical Magnetic Resonance Research, University of Oxford, Oxford OX3 9DU, United Kingdom

Received: 26 June 2012 Accepted: 19 December 2012 Published: 28 December 2012

\section{References}

1. Arheden H, Saeed M, Higgins CB, Gao DW, Bremerich J, Wyttenbach R, Dae MW, Wendland MF. Measurement of the distribution volume of gadopentetate dimeglumine at echo-planar MR imaging to quantify myocardial infarction: comparison with 99mTc-DTPA autoradiography in rats. Radiology. 1999; 211:698-708.

2. Moon JC. Myocardial tissue characterisation using gadolinium CMR: University of London Library; 2005. Chapter 12.

3. Ugander M, Oki AJ, Hsu LY, Kellman P, Greiser A, Aletras AH, Sibley CT, Chen MY, Bandettini WP, Arai AE. Extracellular volume imaging by magnetic resonance imaging provides insights into overt and sub-clinical myocardial pathology. Eur Heart J. 2012; 33:1268-78.

4. Flett AS, Sado DM, Quarta G, Mirabel M, Pellerin D, Herrey AS, Hausenloy DJ, Ariti C, Yap J, Kolvekar S, Taylor AM, Moon JC. Diffuse myocardial fibrosis in severe aortic stenosis: an equilibrium contrast cardiovascular magnetic resonance study. Eur Heart J Cardiovasc Imaging. 2012; 13:819-26.

5. Jerosch-Herold M, Sheridan DC, Kushner JD, Nauman D, Burgess D, Dutton D, Alharethi R, Li D, Hershberger RE. Cardiac magnetic resonance imaging of myocardial contrast uptake and blood flow in patients affected with idiopathic or familial dilated cardiomyopathy. Am J Physiol Heart Circ Physiol. 2008; 295:H1234-H42.

6. Sado DF, Banypersad A, White S, Maestrini S, Quarta V, Lachmann G, Murphy R, Mehta E, Hughes A, McKenna D, Taylor W, Hausenloy A, Hawkins D, Elliott P, Moon J. Cardiovascular magnetic resonance measurement of myocardial extracellular volume in health and disease. Heart. 2012; in Press.

7. Banypersad S, Sado D, Flett A, Gibbs SD, Pinney JH, Maestrini V, White SK, Dungu J, Hawkins PN, Moon JC. Cardiac involvement in cardiac AL amyloidosis as measured by equilibrium contrast cardiovascular magnetic resonance [abstract]. JCMR. 2012; 14(suppl 1):P174.

8. Schelbert EB, Testa SM, Meier CG, Ceyrolles WJ, Levenson JE, Blair AJ Kellman P, Jones BL, Ludwig DR, Schwartzman D, Shroff SG, Wong TC. Myocardial extravascular extracellular volume fraction measurement by gadolinium cardiovascular magnetic resonance in humans: slow infusion versus bolus. J Cardiovasc Magn Reson. 2011; 4:13-6.

9. Flett AS, Hayward MP, Ashworth MT, Hansen MS, Taylor AM, Elliott PM, McGregor C, Moon JC. Equilibrium contrast cardiovascular magnetic resonance for the measurement of diffuse myocardial fibrosis: preliminary validation in humans. Circulation. 2010; 122:138-44.

10. White SK, Sado DM, Flett AS, Moon JC. Characterising the myocardial interstitial space: the clinical relevance of non-invasive imaging. Heart 2012; 98:773-9.

11. Messroghli DR, Radjenovic A, Kozerke S, Higgins DM, Sivananthan MU, Ridgway JP. Modified look-locker inversion recovery (MOLLI) for high-resolution T1 mapping of the heart. Magn Reson Med. 2004; 52:141-6.

12. Piechnik SK, Ferreira VM, Dall'Armellina E, Cochlin LE, Greiser A, Neubauer S, Robson MD. Shortened modified look- locker inversion recovery (ShMOLLI) for clinical myocardial T1-mapping at 1.5 And 3 T within a 9 heartbeat breathhold. J Cardiovasc Magn Reson. 2010; 12:69.

13. Messroghli DR, Rudolph A, Abdel-Aty H, Wassmuth R, Kühne T, Dietz R, Schulz-Menger J. Open-source software tool for the generation of relaxation time maps in magnetic resonance imaging. BMC Med Imaging. 2010; 10:16.

14. Lee JJ, Liu S, Nacif MS, Ugander M, Han J, Kawel N, Sibley CT, Kellman P, Arai AE, Bluemke DA. Myocardial T1 and extracellular volume fraction mapping at 3 Tesla. J Cardiovasc Magn Reson. 2011; 28:13-75.

15. Xue H, Shah S, Greiser A, Guetter C, Littmann A, Jolly MP, Arai AE, Zuehlsdorff S, Guehring J, Kellman P. Motion correction for myocardial T1 mapping using image registration with synthetic image estimation. Magn Reson Med. 2012; 67:1644-55.

16. Kawel N, Nacif M, Zavodni A, Jones J, Liu S, Sibley CT, Bluemke DA. T1 Mapping of the myocardium: intra-individual assessment of the effect of field strength, cardiac cycle and variation by myocardial region. J Cardiovasc Magn Reson. 2012;1;14(1):27.

17. Kawel N, Marcelo N, Anna Z, Jacquin J, Songtao L, Sibley CT, Bluemke DA. T1 Mapping of the myocardium: intra-individual assessment of post-contrast T1 time evolution and extracellular volume fraction at 3T for Gd-DTPA and Gd-BOPTA. J Cardiovasc Magn Reson. 2012; 14(issue number):26. doi:10.1186/ 1532-429X-14-26.

18. Chow K, Flewitt JA, Pagano JJ, Green JD, Friedrich MG, Thompson RB. MOLLI $\mathrm{T} 1$ values have systematic $\mathrm{T} 2$ and inversion efficiency dependent errors. http://www.medical.siemens.com/siemens/en_INT/gg_mr_FBAs/files/ MAGNETOM_World/ismrm_proceedings/ismrm_2012/395_ISMRM2012.pdf.

doi:10.1186/1532-429X-14-88

Cite this article as: Fontana et al:: Comparison of T1 mapping techniques for ECV quantification. Histological validation and reproducibility of ShMOLLI versus multibreath-hold T1 quantification equilibrium contrast CMR. Journal of Cardiovascular Magnetic Resonance 2012 14:88.

\section{Submit your next manuscript to BioMed Central and take full advantage of:}

- Convenient online submission

- Thorough peer review

- No space constraints or color figure charges

- Immediate publication on acceptance

- Inclusion in PubMed, CAS, Scopus and Google Scholar

- Research which is freely available for redistribution 